EPJ Web of Conferences 106, 00008 (2016)

DOI: $10.1051 /$ epjconf/201610600008

(C) Owned by the authors, published by EDP Sciences, 2016

\title{
Workshop 3 - Reactor Surveillance and Plant Life
}

\author{
Co-chairs: \\ EWGRD: Christophe Destouches (CEA Cadarache, France) \\ ASTM: Arnold Fero (Westinghouse Electric Co, US)
}

- This workshop had 26 participants.

- A copy of a new ASTM Standard (E2956-14, "Standard Guide for Monitoring the Neutron Exposure of LWR Reactor Pressure Vessels") was routed for information. This standard discusses the reasons for performing monitoring, discusses the advantages and disadvantages of various monitoring methods and discusses the international acceptance and experience with the various methods. The standard also includes many "best practices" with regard to installing EVND. A copy of this new standard may be downloaded from http://www.astm.org/ at a cost of \$48 USD.

- Ex-Vessel Neutron Dosimetry (EVND) has become the international norm for monitoring the neutron exposure distribution of the reactor pressure vessel (RPV).

- In South Korea the Korea Atomic Energy Research Institute (KAERI) has implemented EVND in all of the Korean PWRs. The newest reactors will have dosimetry in place from initial reactor startup. They are exporting the method to the four KSNP plants being built in the UAE. EVND is typically evaluated with each surveillance capsule withdrawal and every three fuel cycles otherwise. EVND is strongly approved by the regulator (KINS).

- Vattenfall has installed EVND in their three PWRs and one BWR. They have done significant core design changes to reduce the neutron exposure rate of critical RPV materials. The changes have been confirmed by EVND and RPV clad sampling. Their regulator appreciated the measured confirmation of analytical predictions.

- EVND is in routine use in Russian VVERs. Use of EVND is a regulatory "rule." On the VVER440's RPV clad sampling has also been performed at the same time as EVND evaluation. On the VVERs, evaluation of the neutron exposure of the RPV nozzles has been done by calculation, but no measurements yet.

- KAERI and Westinghouse both commented on the advantages of having large amounts of measured data on similar reactors. This allows much more informed evaluation of reactorspecific measurements and calculations.

- EDF commented that they have limited experience with EVND. They do not have any EVND currently installed and have not forecasted an installation. Nevertheless a re-evaluation of the use of EVND based on the international experience could be envisaged.

- Westinghouse and the US nuclear power plant fleet are using EVND widely following the recommendations of US NRC Regulatory Guide 1.190. Westinghouse shared important

This is an Open Access article distributed under the terms of the Creative Commons Attribution License 2.0, which permits unrestricted use, distribution, and reproduction in any medium, provided the original work is properly cited. 


\section{EPJ Web of Conferences}

feedback regarding the installation of EVND in older power plants with regard to minimizing radiation exposure.

- Parallel in-vessel and ex-vessel dosimetry measurements are recommended.

France is the only country where there is an end-of-life regulatory limit for the reactor vessel neutron fluence.

- Having good RPV material properties results in less regulatory scrutiny.

- Gamma ray flux evaluations:

- There is a need for gamma ray dose rate measurements in the reactor cavity. This relates to aging evaluations of the concrete biological shield in the context of increasing plant licenses from 60 years to 80 or 100 years. Through EVND programs there is a lot of neutron data available.

- Gamma ray energy deposition rates have to be reevaluated in order to optimize the thermohydraulic calculations.

- There is a need for a high dose gamma ray measurement method. One device mentioned was lithium fluoride optical crystals which can provide data in the range of $10^{8} \mathrm{~Gy}$. There needs to be some reference irradiations in this dose range to identify limitations in the optical absorbance measurement with this infrared color center. Reference irradiations are also needed to identify the neutron sensitivity of the depleted lithium fluoride.

- Another device proposed was some sort of integrating "radiometric monitor" that would perform in a similar manner to a neutron activation foil, but where the reaction was induced by gamma ray exposure.

- Fission spectrum:

- The knowledge of the fission spectrum above $5 \mathrm{MeV}$ is important for a good evaluation of the ex-vessel neutron field.

- Studies performed by CEA and Westinghouse indicate that nuclear data libraries can induce up to a $10 \%$ variation in some in-vessel and/or ex-vessel dosimetry reactions.

- Benchmark for reactor surveillance:

- ORNL PCA, VENUS 1, and H.B. Robinson 2 from the SINBAD database are the main validation benchmarks for neutron fluence computation codes.

- New benchmark evaluations are welcomed/needed for SINBAD. 